Two-grid Methods for Mixed Finite Element Approximations of Nonlinear Parabolic Equations

\author{
Clint N. Dawson
}

Mary F. Wheeler

January 1994

TR94-01 



\title{
TWO-GRID METHODS FOR MIXED FINITE ELEMENT APPROXIMATIONS OF NONLINEAR PARABOLIC EQUATIONS
}

\author{
CLINT N. DAWSON AND MARY F. WHEELER
}

\begin{abstract}
Mixed finite element approximation of nonlinear parabolic equations is discussed. The equation considered is a prototype of a model which arises in flow through porous media. A two-grid approximation scheme is developed and analyzed for implicit time discretizations. In this approach, the full nonlinear system is solved on a "coarse" grid of size $H$. The nonlinearities are expanded about the coarse grid solution, and the resulting linear but nonsymmetric system is solved on a "fine" grid of size $h$. Error estimates are derived which demonstrate that the error is $\mathcal{O}\left(h^{k+1}+H^{2(k+1)-d / 2}+\Delta t\right)$, where $k$ is the degree of the approximating space for the primary variable and $d$ is spatial dimension, with $k \geq 1$ for $d \geq 2$. For the $R T_{0}$ space $(k=0)$ on rectangular domains, we present a modified scheme for treating the coarse grid problem. Here we show that the error is $\mathcal{O}\left(h+H^{3-d / 2}+\Delta t\right)$. The above estimates are useful for determining an appropriate $H$ for the coarse grid problem.
\end{abstract}

\section{INTRODUCTION}

In this paper, we consider the mixed finite element approximation of $\rho$ satisfying

$$
\frac{\partial \rho}{\partial t}-\nabla \cdot(K(\rho) \nabla \rho)=f(x, t), \quad \text { on } \Omega \times(0, T]
$$

with initial condition

$$
\rho(x, 0)=\rho^{0}(x), \quad \text { on } \Omega,
$$

and boundary condition

$$
(K(\rho) \nabla \rho) \cdot \nu=0, \text { on } \partial \Omega \times(0, T]
$$

where $\Omega \subset I R^{d}, d \leq 3$, is a bounded, convex domain with $C^{1}$ boundary $\partial \Omega, \nu$ is the unit exterior normal to $\partial \Omega$, and $K$ is a symmetric positive definite tensor and $K: \Omega \times I R \rightarrow I R^{d \times d}$. We further assume that there exist positive constants $K_{*}$ and $K^{*}$ such that for $z \in I R^{d}$

$$
K_{*}\|z\|^{2} \leq z^{t} K(x, s) z \leq K^{*}\|z\|^{2} \text {, for } x \in \Omega \text { and } s \in I R \text {, }
$$

1991 Mathematics Subject Classification. Primary 65M60, 65P05 .

Key words and phrases. Parabolic equations, mixed finite element methods.

This work supported by grants from the Department of Energy and the State of Texas.

The final version of this paper will be submitted for publication elsewhere. 
and that each element of $K$ is twice continuously differentiable with derivatives up to second order bounded above by $K^{*}$.

Equation (1) is a simplification of a nonlinear parabolic equation (Ricliard's Equation) which arises in the modeling of two-phase flow in porous media, where the two phases are air and water [3]. The major difference between Richards' Equation and (1) is in the time derivative term, where $\rho$ is replaced by a nonlinear function $\theta(\rho)$. The function $\theta^{\prime}(\rho)$ may be zero and this complicates the analysis; however, this difficulty may be handled by techniques given in [1]. For brevity, we do not consider this generalization here.

Let $L^{p}(\Omega)$ for $p \geq 1$ denote the standard Banach space defined on $\Omega$, with norm $\|\cdot\|_{p}$. We shall denote by $W^{m, p}(\Omega)$ the standard Sobolev space ( $m$ partial derivatives in $L^{p}$ ) with norm $\|\cdot\|_{m, p}$. The $W^{m, p}$ norm for vectors will be understood to be for each component. When $p=2$ we omit the subscript on the norms.

Let $(\cdot, \cdot)$ denote the $L^{2}(\Omega)$ inner product, scalar and vector. Let $V=H($ div $; \Omega)=$ $\left\{v \in\left(L^{2}(\Omega)\right)^{d}: \nabla \cdot v \in L^{2}(\Omega)\right\}, \tilde{V}=V \cap\{v \cdot \nu=0\}$, and $W=L^{2}(\Omega)$.

As in [2] we define the mixed weak form of $(1)$ as $(\rho, \Gamma, \Psi) \in W \times V \times \tilde{V}$ satisfying

$$
\begin{gathered}
\left(\frac{\partial \rho}{\partial t}, w\right)+(\nabla \cdot \Psi, w)=(f, w), \quad w \in W, \\
(\Gamma, v)-(\rho, \nabla \cdot v)=0, \quad v \in \tilde{V}, \\
(\Psi, v)=(K(\rho) \Gamma, v), \quad v \in V .
\end{gathered}
$$

We consider finite-dimensional subspaces $W_{h}^{k}, \tilde{V}_{h}^{k}$, and $V_{h}^{k}$ of $W, \tilde{V}$, and $V$, respectively (they may be Raviart-Thomas-Nedelec spaces of index $k R T_{k}[9,10]$ or Brezzi-Douglas-Marini spaces of index $k$ [4] for instance) associated with a quasiuniform family of polygonal decompositions of $\Omega$ by triangles (tetrahedra) or bricks with diameter $h$. For simplicity in the discussion below, we will drop the superscript $k$.

Let $N>0$ and $\triangle t=T / N, t^{n}=n \triangle t$, and $\varphi^{n}=\varphi\left(\cdot, t^{n}\right)$. In our analysis we shall use the following norms: for $X$ a normed vector space defined on $\Omega$,

$$
\begin{gathered}
\|\varphi\|_{l^{2}((0, T) ; X)} \equiv\left(\sum_{n=1}^{N} \Delta t\left\|\varphi^{n}\right\|_{X}^{2}\right)^{\frac{1}{2}}, \\
\|\varphi\|_{l \infty((0, T) ; X)} \equiv \max _{1 \leq n \leq N}\left\|\varphi^{n}\right\|_{X},
\end{gathered}
$$

and

$$
\|\varphi\|_{L^{2}((0, T) ; X)} \equiv\left(\int_{0}^{T}\left\|\varphi^{n}(\cdot, t)\right\|_{X}^{2} d t\right)^{\frac{1}{2}} .
$$

The discrete time mixed finite element approximation to (5)-(7) is defined as follows: Given $\left(\rho_{h}^{0}, \Gamma_{h}^{0}, \Psi_{h}^{0}\right) \in W_{h} \times V_{h} \times \tilde{V}_{h}$, for $n=1, \ldots, N$, let $\left(\rho_{h}^{n}, \Gamma_{h}^{n}, \Psi_{h}^{n}\right) \in$ $W_{h} \times V_{h} \times \tilde{V}_{h}$, satisfy

$$
\begin{gathered}
\left(\frac{\rho_{h}^{n}-\rho_{h}^{n-1}}{\Delta t}, w_{h}\right)+\left(\nabla \cdot \Psi_{h}^{n}, w_{h}\right)=\left(f^{n}, w_{h}\right), \quad w_{h} \in W_{h}, \\
\left(\Gamma_{h}^{n}, v_{h}\right)-\left(\rho_{h}^{n}, \nabla \cdot v_{h}\right)=0, \quad v_{h} \in \tilde{V}_{h}
\end{gathered}
$$




$$
\left(\Psi_{h}^{n}, v_{h}\right)=\left(K\left(\rho_{h}^{n}\right) \Gamma_{h}^{n}, v_{h}\right), \quad v_{h} \in V_{h} .
$$

This procedure is based on a modification of the standard mixed finite element procedure and was introduced for linear elliptic problems in [2]. For brevity, a proof of existence and uniqueness of the solution to the nonlinear algebraic system (8)-(10) is not given; the reader is referred to [7] since the proof closely follows the argument of Milner for standard mixed method approximations to nonlinear elliptic problems.

Instead of solving (8)-(10) each time step for a fine mesh $h$ we shall also consider two two-level procedures, both of which involve solving the nonlinear problem only a coarse grid of diameter $H>>h$. This work has been motivated by the work of $\mathrm{Xu}$ [12] for Galerkin procedures applied to nonlinear elliptic equations. In the simplest scheme, the fine mesh discrete problem is linearized by evaluating $K(\rho)$ at the coarse mesh solution $\rho_{H}$. In the second scheme $K(\rho)$ is approximated by a first order Taylor expansion about $\rho_{H}$, a correction by one Newton iteration on the fine space. We shall show that the simplest scheme yields no improvement in accuracy over the coarse grid approximation. The second scheme; however, yields substantial improvement and is a viable computational approach.

This paper is divided into five sections. Notation and approximation assumptions are given and error estimates are derived for (8)-(10) in Section 2. The two twolevel schemes are defined and estimates derived for both in Section 3. A new procedure based on postprocessing the coarse grid solution and then applying a Newton correction is defined and analyzed in Section 4 for the space $R T_{0}$. In Section 5, we give conclusions and extensions.

\section{Notation and Approximation Results}

We shall employ several projection operators.

Denote by $P$ the $L^{2}$ projection operator. More precisely, let $P_{W_{h}}: L^{2}(\Omega) \rightarrow W_{h}$ and $P_{V_{h}}:\left(L^{2}(\Omega)\right)^{d} \rightarrow V_{h}$, where for $g \in L^{2}(\Omega)$,

$$
\left(P_{W_{h}} g, w_{h}\right)=\left(g, w_{h}\right), \quad w_{h} \in W_{h},
$$

and for $q \in\left(L^{2}(\Omega)\right)^{d}$,

$$
\left(P_{V_{h}} q, v_{h}\right)=\left(q, v_{h}\right), \quad v_{h} \in V_{h} .
$$

For convenience we shall set $\hat{g}_{h}=P g$, where $P$ is understood to be $P_{W_{h}}$ or $P_{V_{h}}$ depending on whether $g$ is a scalar or vector quantity.

We shall use the well known $\Pi_{h}$ projection for mixed funite element approximation spaces. We shall assume that there exists a projection operator $\Pi_{h}$ : $\left(H^{1}(\Omega)\right)^{d} \rightarrow V_{h}$ such that

$$
\left(\nabla \cdot \Pi_{h} g, w_{h}\right)=\left(\nabla \cdot g, w_{h}\right), \quad w_{h} \in W_{h} .
$$

We assume that

$$
\nabla \cdot \Pi_{h}=P_{W_{h}}, \quad \nabla \cdot: V \stackrel{\text { onto }}{\longrightarrow} W_{h} .
$$

We also assume that the following approximation properties hold. 
Approximation Properties A:

- There exists a positive constant $Q$ independent of $h$ such that

$$
\begin{gathered}
\left\|u-\Pi_{h} u\right\| \leq Q\|u\|_{r} h^{r}, \quad 1 \leq r \leq k+1, \\
\left\|p-\hat{p}_{h}\right\| \leq Q\|p\|_{r} h^{r}, \quad 0 \leq r \leq k+1,
\end{gathered}
$$

and

$$
\left\|p-\hat{p}_{h}\right\|_{\infty} \leq Q\|p\|_{\infty} h^{r}, \quad 0 \leq r \leq k+1 .
$$

- Given $\Upsilon \in\left(W^{1, \infty}(\Omega)\right)^{d}$ there exists a $\Upsilon_{h} \in V_{h}$ such that

$$
\left\|\Upsilon-\Upsilon_{h}\right\| \leq Q h\|\Upsilon\|_{1, \infty} .
$$
$V_{h}$,

Finally, we assume the following inverse property on $V_{h}$ holds, namely, for $v_{h} \in$

$$
\left\|v_{h}\right\|_{\infty} \leq\left\|v_{h}\right\| h^{-d / 2} .
$$

These assumptions are known to hold for the Raviart-Thomas-Nedelec and BrezziDouglas-Marini spaces mentioned above.

In this paper $\mathrm{C}$ shall denote a generic constant. For convenience we will also assume that the solution is smooth and that the maximum index of convergence $k+1$ is attained.

We rewrite (5)-(7) with $t=t^{n}$. Using the definition of $\hat{\rho}$, the $\Pi_{h}$ projection, and the assumption that $\nabla \cdot V_{h} \subset W_{h}$, we deduce that

$$
\begin{gathered}
\left(\frac{\hat{\rho}_{h}^{n}-\hat{\rho}_{h}^{n-1}}{\Delta t}, w_{h}\right)+\left(\nabla \cdot \Pi_{h} \Psi^{n}, w_{h}\right)=\left(f^{n}, w_{h}\right)+\left(\epsilon^{n}, w_{h}\right), \quad w_{h} \in W_{h}, \\
\left(\hat{\Gamma}_{h}^{n}, v_{h}\right)-\left(\hat{\rho}_{h}^{n}, \nabla \cdot v_{h}\right)=0, \quad v_{h} \in \tilde{V}_{h},
\end{gathered}
$$

and

$$
\left(\Pi_{h} \Psi^{n}, v_{h}\right)=\left(\Pi_{h} \Psi^{n}-\Psi^{n}, v_{h}\right)+\left(K\left(\rho^{n}\right) \Gamma^{n}, v_{h}\right), \quad v_{h} \in V_{h},
$$

where $\epsilon^{n}$ is a time truncation error of order $\Delta t$.

We now derive an error estimate for the algorithm (8)-(10).

Set $\mu^{n}=\hat{\rho}_{h}^{n}-\rho_{h}^{n}, \zeta^{n}=\hat{\Gamma}_{h}^{n}-\Gamma_{h}^{n}$, and $\chi^{n}=\Pi_{h} \Psi^{n}-\Psi_{h}^{n}$. Subtracting (8) from (19), (9) from (20), and (10) from (21) and in the resulting equations using the test functions $w_{h}=\mu^{n}, v_{h}=\chi^{n}$, and $v_{h}=\zeta^{n}$ in the first, second, and third equations, respectively, we obtain the error equations

$$
\begin{gathered}
\left(\frac{\mu^{n}-\mu^{n-1}}{\Delta t}, \mu^{n}\right)+\left(\nabla \cdot \chi^{n}, \mu^{n}\right)=\left(\epsilon^{n}, \mu^{n}\right), \\
\left(\zeta^{n}, \chi^{n}\right)=\left(\mu^{n}, \nabla \cdot \chi^{n}\right),
\end{gathered}
$$

and

$$
\begin{aligned}
\left(\chi^{n}, \zeta^{n}\right)= & \left(\Pi_{h} \Psi^{n}-\Psi^{n}, \zeta^{n}\right)+\left(K\left(\rho_{h}^{n}\right) \zeta^{n}, \zeta^{n}\right) \\
& -\left(\left(K\left(\rho_{h}^{n}\right)-K\left(\rho^{n}\right)\right) \Gamma^{n}, \zeta^{n}\right) \\
& +\left(K\left(\rho_{h}^{n}\right)\left(\Gamma^{n}-\hat{\Gamma}_{h}^{n}\right), \zeta^{n}\right) .
\end{aligned}
$$


Combining (22) - (24), applying smoothness and boundedness assumptions on $K(\rho)$, and Hölder's inequality we obtain

$$
\begin{aligned}
\frac{1}{2 \triangle t}[ & {\left[\left\|\mu^{n}\right\|^{2}-\left\|\mu^{n-1}\right\|^{2}\right]+\left\|K\left(\rho_{h}^{n}\right)^{1 / 2} \zeta^{n}\right\|^{2} } \\
\leq & \left(\frac{\mu^{n}-\mu^{n-1}}{\Delta t}, \mu^{n}\right)+\left\|K\left(\rho_{h}^{n}\right)^{1 / 2} \zeta^{n}\right\|^{2} \\
\leq & \frac{1}{2}\left\|\mu^{n}\right\|^{2}+\frac{1}{2}\left\|\epsilon^{n}\right\|^{2}+\left(\Pi_{h} \Psi^{n}-\Psi^{n}, \zeta^{n}\right) \\
& +\left(\left(K\left(\rho_{h}^{n}\right)-K\left(\rho^{n}\right)\right) \Gamma^{n}, \zeta^{n}\right)+\left(K\left(\rho_{h}^{n}\right)\left(\Gamma^{n}-\hat{\Gamma}_{h}^{n}\right), \zeta^{n}\right) \\
\leq & \frac{1}{2}\left\|\mu^{n}\right\|^{2}+\frac{1}{2}\left\|\epsilon^{n}\right\|^{2}+\delta\left\|\zeta^{n}\right\|^{2} \\
& +C\left[\left\|\Pi_{h} \Psi^{n}-\Psi^{n}\right\|^{2}+\left\|\hat{\Gamma}_{h}^{n}-\Gamma^{n}\right\|^{2}+\left(\left\|\rho^{n}-\hat{\rho}_{h}^{n}\right\|\left\|\Gamma^{n}\right\|_{\infty}\right)^{2}\right]
\end{aligned}
$$

where $\delta \leq K_{*}$. Multiplying (26) by $\Delta t$ and summing on $n, n=1,2, \ldots, N$ and applying Gronwall's Lemma, we see that

$$
\begin{aligned}
\left\|\mu^{N}\right\|^{2} & +\sum_{n=1}^{N} \Delta t\left\|K\left(\rho_{h}^{n}\right)^{1 / 2} \zeta^{n}\right\|^{2} \\
\leq & C \sum_{n=1}^{N} \Delta t\left[\left\|\Pi_{h} \Psi^{n}-\Psi^{n}\right\|^{2}+\left\|\hat{\Gamma}_{h}^{n}-\Gamma^{n}\right\|^{2}+\left(\left\|\rho^{n}-\hat{\rho}_{h}^{n}\right\|\left\|\Gamma^{n}\right\|_{\infty}\right)^{2}\right] \\
& +\left\|\mu^{0}\right\|^{2}+C \triangle t^{2} .
\end{aligned}
$$

Using (27), Approximation Properties A, and the triangle inequality we deduce the following theroem:

Theorem 1. Let $V_{h}=V_{h}^{k}, \tilde{V}_{h}=\tilde{V}_{h}^{k}$, and $W_{h}=W_{h}^{k}$ and define the triplet $\left(\rho_{h}^{n}, \Gamma_{h}^{n}, \Psi_{h}^{n}\right) \in W_{h}^{k} \times V_{h}^{k} \times \tilde{V}_{h}^{k} b y(8)-(10)$, for $n \geq 1$. Assume that the Approximation Properties $A$ hold. Take $\rho_{h}^{0}=\hat{\rho}_{h}(\cdot, 0)$. Then there exists a positive constant $C$, independent of $h$ such that

$$
\left\|\rho_{h}^{N}-\rho^{N}\right\|+\left(\sum_{n=1}^{N} \Delta t\left\|K\left(\rho_{h}^{n}\right)^{1 / 2}\left(\Gamma_{h}^{n}-\Gamma^{n}\right)\right\|^{2}\right)^{\frac{1}{2}} \leq C\left(h^{k+1}+\Delta t\right) .
$$

\section{Two-Level Schemes}

We first consider a scheme based on a correction by one Newton iteration on the fine space. More precisely, we solve (8)-(10) on a coarse mesh with $h=H$ and then solve the following linear system for $\left(\tilde{\rho}_{h}^{n}, \tilde{\Gamma}_{h}^{n}, \tilde{\Psi}_{h}^{n}\right) \in W_{h} \times V_{h} \times \tilde{V}_{h}$, for $n \geq 1$ and $h<<H$ :

$$
\begin{gathered}
\left(\frac{\tilde{\rho}_{h}^{n}-\tilde{\rho}_{h}^{n-1}}{\Delta t}, w_{h}\right)+\left(\nabla \cdot \tilde{\Psi}_{h}^{n}, w_{h}\right)=\left(f^{n}, w_{h}\right), \quad w_{h} \in W_{h}, \\
\left(\tilde{\Gamma}_{h}^{n}, v_{h}\right)=\left(\tilde{\rho}_{h}^{n}, \nabla \cdot v_{h}\right), \quad v_{h} \in \tilde{V}_{h}, \\
\left(\tilde{\Psi}_{h}^{n}, v_{h}\right)=\left(K\left(\rho_{H}^{n}\right) \tilde{\Gamma}_{h}^{n}, v_{h}\right)+\left(K^{\prime}\left(\rho_{H}^{n}\right) \Gamma_{H}^{n}\left(\tilde{\rho}_{h}^{n}-\rho_{H}^{n}\right), v_{h}\right), \quad v_{h} \in V_{h} .
\end{gathered}
$$


Equation (30) is motivated by the Taylor expansion

$$
K\left(\rho^{n}\right) \Gamma^{n}=K\left(\rho_{H}^{n}\right) \Gamma^{n}+K^{\prime \prime}\left(\rho_{H}^{n}\right) \Gamma^{n}\left(\rho^{n}-\rho_{H}^{N}\right)+\frac{K^{\prime \prime}\left(\alpha^{n}\right)}{2} \Gamma^{n}\left(\rho^{n}-\rho_{H}^{n}\right)^{2},
$$

for some $\alpha^{n}$ between $\rho^{n}$ and $\rho_{H}^{n}$.

We now derive an estimate for this two-level scheme. Set $\xi^{n}=\hat{\rho}_{h}^{n}-\tilde{\rho}_{h}^{n}, v^{n}=$ $\hat{\Gamma}_{h}^{n}-\tilde{\Gamma}_{h}^{n}$, and $\theta^{n}=\Pi_{h} \Psi^{n}-\tilde{\Psi}_{h}^{n}$.

Subtracting (28) from (19), (29) from (20), and (30) from (21), letting $w_{h}=\xi^{n}$, $v_{h}=\theta^{n}$, and $v_{h}=v^{n}$, in the first, second, and third equations respectively, we obtain

$$
\begin{gathered}
\left(\frac{\xi^{n}-\xi^{n-1}}{\Delta t}, \xi^{n}\right)+\left(\nabla \cdot \theta^{n}, \xi^{n}\right)=\left(\epsilon^{n}, \xi^{n}\right) \\
\left(v^{n}, \theta^{n}\right)=\left(\xi^{n}, \nabla \cdot \theta^{n}\right),
\end{gathered}
$$

and

$$
\begin{aligned}
\left(\theta^{n}, v^{n}\right)=( & \left.\Pi_{h} \Psi^{n}-\Psi^{n}, v^{n}\right)-\left(K\left(\rho^{n}\right)\left(\hat{\Gamma}_{h}^{n}-\Gamma^{n}\right), v^{n}\right) \\
& +\left(K\left(\rho_{H}^{n}\right) v^{n}, v^{n}\right)+\left(\left(\xi^{n}+\left(\rho^{n}-\hat{\rho}_{h}^{n}\right)\right) K^{\prime \prime}\left(\rho_{H}^{n}\right) \Gamma_{H}^{n}, v^{n}\right) \\
& +\left(\left(K^{\prime}\left(\rho_{H}^{n}\right)\left(\rho^{n}-\rho_{H}^{n}\right)\left(\hat{\Gamma}_{h}^{n}-\Gamma_{H}^{n}\right), v^{n}\right)\right. \\
& +\frac{1}{2}\left(\left(\rho^{n}-\rho_{H}^{n}\right)^{2} K^{\prime \prime}\left(\alpha^{n}\right) \hat{\Gamma}_{h}^{n}, v^{n}\right) .
\end{aligned}
$$

Combining (31)-(34) and using Hölder's inequality, we deduce that

$$
\begin{aligned}
& \frac{1}{2 \Delta t}\left[\left\|\xi^{n}\right\|^{2}-\left\|\xi^{n-1}\right\|^{2}\right]+\left\|K\left(\rho_{H}^{n}\right)^{1 / 2} v^{n}\right\|^{2} \\
& \leq\left|\left(\xi^{n}, \epsilon^{n}\right)\right|+\delta\left\|v^{n}\right\|^{2} \\
& +C\left\|v^{n}\right\|\left\|\xi^{n}\right\|\left(\left\|\Gamma^{n}\right\|_{\infty}+\left\|\left(\Gamma_{H}^{n}-\Gamma^{n}\right)\right\|_{\infty}\right) \\
& +C\left[\left\|\Pi_{h} \Psi^{n}-\Psi^{n}\right\|^{2}+\left\|\Gamma_{h}^{n}-\Gamma^{n}\right\|^{2}\right. \\
& +\left\|\left(\rho^{n}-\hat{\rho}_{h}^{n}\right) \Gamma_{H}^{n}\right\|^{2} \\
& +\left\|\left(\rho^{n}-\rho_{H}^{n}\right)\left(\hat{\Gamma}_{h}^{n}-\Gamma_{H}^{n}\right)\right\|^{2} \\
& + \\
& \left.+\left\|\left(\rho^{n}-\rho_{H}^{n}\right)^{2} \hat{\Gamma}_{h}^{n}\right\|^{2}\right],
\end{aligned}
$$

where $\delta \leq K_{*} / 2$, and $C=C\left(K^{*}, K_{*}\right)$.

We now choose $\hat{N}$ to be the index corresponding to the maximum of $\left\|\xi^{n}\right\|$ for $n=1, \ldots, N$. Multiplying (35) by $\Delta t$ and summing from $n=1$ to $M, M \leq N$, using Approximation Properties A and Theorem 1, we see that

$$
\begin{aligned}
& \left\|\xi^{M}\right\|^{2}-\left\|\xi^{0}\right\|^{2}+\sum_{n=1}^{M} \Delta t\left\|K\left(\rho_{H}^{n}\right)^{1 / 2} v^{n}\right\|^{2} \\
& (35) \leq C \triangle t^{2}\left\|\rho_{t}\right\|_{L^{2}\left((0, T), L^{2}(\Omega)\right)}^{2}+\delta \sum_{n=1}^{M} \Delta t\left\|v^{n}\right\|^{2}+T^{*}+T^{* *} \\
& \quad+C\left[\sum_{n=1}^{M} \Delta t\left\|\xi^{n}\right\|^{2}+\|\Psi\|_{l^{2}\left((0, T), H^{k+1}\right)}^{2} h^{2(k+1)}+\|\Gamma\|_{l^{2}\left((0, T), H^{k+1}\right)}^{2} h^{2(k+1)}\right],
\end{aligned}
$$


where $T^{*}$ and $T^{* *}$ are defined as follows.

First, by Theorem 1 and the inverse assumption (18)

$$
\begin{aligned}
T^{*} & =K^{*}\left\|\xi^{\hat{N}}\right\|\left(\sum_{n=1}^{M} \Delta t\left\|v^{n}\right\|^{2}\right)^{\frac{1}{2}}\left(\sum_{n=1}^{M} \Delta t\left\|\left(\Gamma_{H}^{n}-\hat{\Gamma}_{H}^{n}\right)\right\|_{\infty}^{2}\right)^{\frac{1}{2}} \\
& \leq K^{*}\left\|\xi^{\hat{N}}\right\|\left(\sum_{n=1}^{M} \Delta t\left\|v^{n}\right\|^{2}\right)^{\frac{1}{2}}\left(H^{-d / 2}\left(H^{k+1}+\triangle t\right)\right)
\end{aligned}
$$

For $d \geq 2, k \geq 1, H$ and $\Delta t$ can be chosen sufficiently small so that

$$
T^{*} \leq \frac{1}{2}\left\|\xi^{\hat{N}}\right\|^{2}+\frac{K_{*}}{8} \sum_{n=1}^{N} \Delta t\left\|v^{n}\right\|^{2} .
$$

Moreover,

$$
\begin{aligned}
T^{* *}=C^{*} & \sum_{n=1}^{M}\left(\left\|\left(\rho^{n}-\hat{\rho}_{h}^{n}\right) \Gamma_{H}^{n}\right\|^{2}+\left\|\left(\rho^{n}-\rho_{H}^{n}\right)\left(\hat{\Gamma}_{h}^{n}-\Gamma_{H}^{n}\right)\right\|^{2}\right. \\
& \left.+\left\|\left(\rho^{n}-\rho_{H}^{n}\right)^{2} \hat{\Gamma}_{h}^{n}\right\|^{2}\right) \triangle t \\
\leq \quad C^{*} & \sum_{n=1}^{M} \Delta t\left(\left\|\rho^{n}-\hat{\rho}_{h}^{n}\right\|^{2}\left\|\Gamma^{n}\right\|_{\infty}^{2}\right. \\
& +\left\|\rho^{n}-\hat{\rho}_{h}^{n}\right\|_{\infty}^{2}\left\|\Gamma_{H}^{n}-\Gamma^{n}\right\|^{2} \\
& +\left(\left\|\hat{\rho}_{H}^{N}-\rho_{H}^{N}\right\|_{\infty}+\left\|\rho^{N}-\hat{\rho}_{H}^{N}\right\|_{\infty}\right)^{2}\left\|\hat{\Gamma}_{h}^{n}-\Gamma_{H}^{n}\right\|^{2} \\
& \left.+\left\|\rho^{n}-\rho_{H}^{n}\right\|_{\infty}^{2}\left\|\rho^{n}-\rho_{H}^{n}\right\|^{2}\left\|\hat{\Gamma}_{h}^{n}\right\|_{\infty}^{2}\right) .
\end{aligned}
$$

Thus, from Theorem 1 and approximation properties,

$$
\begin{gathered}
T^{* *} \leq C^{*} \sum_{n=1}^{M} \Delta t\left[h^{2(k+1)}+\left(H^{-d / 2}\left(H^{k+1}+\Delta t\right)+H^{k+1}\right)^{2}\left(H^{k+1}+\Delta t\right)^{2}\right. \\
\left.+\left(H^{-d / 2}\left(H^{k+1}+\Delta t\right)\right)^{2}\left(H^{k+1}+\Delta t\right)^{2}\right] .
\end{gathered}
$$

Here $C^{*}$ depends on $\|\rho\|_{l^{2}\left((0, T) ; W^{(k+1), \infty}\right)},\|\Gamma\|_{l^{2}\left((0, T) ; W^{1, \infty}\right)},\|\Gamma\|_{l^{2}\left((0, T) ; H^{(k+1)}\right)}, K^{*}$ and $K_{*}$.

Taking $M=\hat{N}$, and for $d \geq 2$ taking $k \geq 1$, noting that $\xi^{0}=0$, and applying Gronwall's Lemma to (36) we see that for $\Delta t$ and $H$ sufficiently small,

$$
\left\|\xi^{\hat{N}}\right\| \leq C\left(h^{k+1}+\Delta t+H^{2 k+2-d / 2}\right) .
$$

Combining (36) - (39), taking $M=N$, we deduce that

$$
\begin{aligned}
\left\|\xi^{N}\right\| & +\left(\sum_{n=1}^{N} \Delta t\left\|K\left(\rho_{H}^{n}\right)^{1 / 2} v^{n}\right\|^{2}\right)^{\frac{1}{2}} \\
& \leq C\left(C^{*}\right)\left(h^{k+1}+\Delta t+H^{2 k+2-d / 2}\right) .
\end{aligned}
$$

Applying the triangle inequality and approximation properities, we obtain the following theorem: 
Theorem 2. Let $V_{h}=V_{h}^{k}, \tilde{V}_{h}=\tilde{V}_{h}^{k}$, and $W_{h}=W_{h}^{k}$ and define the one Newton correction triplet $\left(\tilde{\rho}_{h}^{n}, \hat{\Gamma}_{h}^{n}, \tilde{\Psi}_{h}^{n}\right), \in W_{h}^{k} \times V_{h}^{k} \times \tilde{V}_{h}^{k}$ by (28)-(30). Assume that the Approximation Properties A holds, and take $\hat{\rho}_{h}^{0}=\hat{\rho}_{h}(\cdot, 0)$. Then there exists a positive constant $C^{* *}$, independent of $h$ such that

$$
\begin{aligned}
\| \tilde{\rho}_{h}^{N} & -\rho^{N} \|+\left(\sum_{n=1}^{N} \Delta t\left\|K\left(\rho_{H}^{n}\right)^{1 / 2}\left(\tilde{\Gamma}_{h}^{n}-\Gamma^{n}\right)\right\|^{2}\right)^{\frac{1}{2}} \\
& \leq C^{* *}\left(h^{k+1}+\Delta t+H^{2 k+2-d / 2}\right) .
\end{aligned}
$$

$C^{* *}$ depends on $\|\rho\|_{l^{2}\left((0, T) ; W^{(k+1), \infty}\right)},\|\Gamma\|_{l^{2}\left((0, T) ; W^{1, \infty}\right)},\|\Gamma\|_{l^{2}\left((0, T), H^{(k+1)}\right)}, K^{*}$ and $K_{*}$.

We now consider the simple two level scheme: solve(8)-(10) on a coarse mesh with $h=H$ and then solve the following linear system for $\left(\bar{\rho}_{h}^{n}, \bar{\Gamma}_{h}^{n}, \bar{\Psi}_{h}^{n}\right) \in W_{h} \times$ $V_{h} \times \tilde{V}_{h}$ for $n \geq 1$,

$$
\begin{gathered}
\left(\frac{\bar{\rho}_{h}^{n}-\bar{\rho}_{h}^{n-1}}{\Delta t}, w_{h}\right)+\left(\nabla \cdot \bar{\Psi}_{h}^{n}, w_{h}\right)=\left(f^{n}, w_{h}\right), \quad w_{h} \in W_{h}, \\
\left(\bar{\Gamma}_{h}^{n}, v_{h}\right)=\left(\bar{\rho}_{h}^{n}, \nabla \cdot v_{h}\right), \quad v_{h} \in \tilde{V}_{h}, \\
\left(\bar{\Psi}_{h}^{n}, v_{h}\right)=\left(K\left(\rho_{H}^{n}\right) \bar{\Gamma}_{h}^{n}, v_{h}\right) \quad v_{h} \in V_{h} .
\end{gathered}
$$

We now derive an estimate for this two-level scheme. Set $\alpha^{n}=\hat{\rho}_{h}^{n}-\bar{\rho}_{h}^{n}, \beta^{n}=$ $\hat{\Gamma}_{h}^{n}-\bar{\Gamma}_{h}^{n}$, and $\lambda^{n}=\Pi_{h} \Psi^{n}-\bar{\Psi}_{h}^{n}$. Subtracting (42) from (19), (43) from (20), and (44) from (21), letting $w_{h}=\alpha^{n}, v_{h}=\lambda^{n}$, and $v_{h}=\beta^{n}$, in the first, second, and third equations respectively, we obtain

$$
\begin{gathered}
\left(\frac{\alpha^{n}-\alpha^{n-1}}{\Delta t}, \alpha^{n}\right)+\left(\nabla \cdot \lambda^{n}, \alpha^{n}\right)=\left(\epsilon^{n}, \alpha^{n}\right) \\
\left(\beta^{n}, \lambda^{n}\right)=\left(\alpha^{n}, \nabla \cdot \lambda^{n}\right),
\end{gathered}
$$

and

$$
\begin{aligned}
\left(\lambda^{n}, \beta^{n}\right)= & \left(\Pi_{h} \Psi^{n}-\Psi^{n}, \beta^{n}\right)-\left(K\left(\rho^{n}\right)\left(\bar{\Gamma}_{h}^{n}-\Gamma^{n}\right), \beta^{n}\right) \\
& +\left(K\left(\rho_{H}^{n}\right) \beta^{n}, \beta^{n}\right)+\left(\left(K\left(\rho^{n}\right)-K\left(\rho_{H}^{n}\right)\right) \hat{\Gamma}_{h}^{n}, \beta^{n}\right) .
\end{aligned}
$$

Combining (45) - (47) we obtain

$$
\begin{aligned}
& \left\|\alpha^{N}\right\|^{2}+\sum_{n=1}^{N} \Delta t\left\|K\left(\rho_{H}^{n}\right)^{\frac{1}{2}} \beta^{n}\right\|^{2} \\
& \quad \leq C\left(\left\|\rho_{H}^{n}-\rho^{n}\right\|^{2}+\left\|\Gamma^{n}-\bar{\Gamma}_{h}^{n}\right\|^{2}+\Delta t^{2}\right) .
\end{aligned}
$$

Theorem 3. The error bound for the simple two level scheme defined by (42) (44) is given by

$$
\begin{gathered}
\left\|\bar{\rho}^{N}-\rho^{N}\right\|+\left(\sum_{n=1}^{N} \Delta t\left\|K\left(\rho_{H}^{n}\right)^{\frac{1}{2}}\left(\check{\Gamma}_{h}^{n}-\Gamma^{n}\right)\right\|\right)^{\frac{1}{2}} \\
\leq C\left(H^{k+1}+\Delta t+h^{k+1}\right) .
\end{gathered}
$$


In the estimate (49) we observe that solving a linear problem on a fine grid with no Newton-type correction yields no asymptotic improvement over a coarse grid solution. This is different from the result obtained for Galerkin methods for nonlinear elliptic equations [12].

\section{NeWton Correction for RT0 Spaces}

Our previous results did not treat the case $k=0$ for $d \geq 2$. Here we assume $\Omega$ is a rectangular parallelepiped, and we restrict our attention to the $R T_{0}(k=0)$ space defined on a tensor product grid. We also assume $K$ is a diagonal matrix or the mesh is uniform.

In this case, the space $W_{h}$ is the space of discontinuous piecewise constants defined on a tensor product partition. The $i^{t h}$ component of the velocity field $V_{h}$ is a continuous piecewise linear polynomial in the $i^{t h}$ direction and discontinuous piecewise constants in the other directions.

The mixed finite element method for the $R T_{0}$ space witl special numerical quadrature rules has been shown [11] to be equivalent to cell centered finite difference methods. The results obtained here are applicable to the latter methods.

A discrete time mixed finite element approximation for the $R T_{0}$ space is defined as follows: Given $\left(\rho_{h}^{0}, \Gamma_{h}^{0}, \Psi_{h}^{0}\right) \in W_{h} \times V_{h} \times \tilde{V}_{h}$, for $n=1, \ldots, N$, let $\left(\rho_{h}^{n}, \Gamma_{h}^{n}, \Psi_{h}^{n}\right) \in$ $W_{h} \times V_{h} \times \tilde{V}_{h}$, satisfy

$$
\begin{gathered}
\left(\frac{\rho_{h}^{n}-\rho_{h}^{n-1}}{\triangle t}, w_{h}\right)+\left(\nabla \cdot \Psi_{h}^{n}, w_{h}\right)=\left(f^{n}, w_{h}\right), \quad w_{h} \in W_{h}, \\
\left(\Gamma_{h}^{n}, v_{h}\right)-\left(\rho_{h}^{n}, \nabla \cdot v_{h}\right)=0, \quad v_{h} \in \bar{V}_{h}, \\
\left(\Psi_{h}^{n}, v_{h}\right)=\left(K\left(\mathcal{P}\left(\rho_{h}^{n}\right)\right) \Gamma_{h}^{n}, v_{h}\right), \quad v_{h} \in V_{h} .
\end{gathered}
$$

Here $\mathcal{P}: W_{h} \rightarrow M_{h}$ is a "postprocessing" operator, where $M_{h}$ denotes the space of continuous bilinears $(d=2)$ or trilinears $(d=3)$ defined on the same partition as the $R T_{0}$ space. For $d=2, \mathcal{P}$ linearly interpolates the four adajacent cell centered values to the vertices of the tensor product grid. A similar procedure can be defined for $d=3$. This postprocessing operator is motivated by well-known superconvergence results for the scalar variable $\rho$ at the center of each grid block $[5,8]$.

In deriving an error estimate for this algorithm we proceed as in Section 2; however here we will employ superconvergence results for the $R T_{k}$ spaces on rectangular elements. It is known for example that the $\Pi_{h}$ and the $P_{V_{h}}$ projections are super close, namely $O\left(h^{k+2}\right)$ in $L^{2}[8,6]$. It is also known that two weighted $L^{2}$ projections are $O\left(h^{k+2}\right)$ provided the weight functions are $\mathcal{C}^{1}$. We shall use these results in the analysis given below.

Set $\mu^{n}=\hat{\rho}_{h}^{n}-\rho_{h}^{n}, \zeta^{n}=\hat{\Gamma}_{h}^{n}-\Gamma_{h}^{n}$, and $\chi^{n}=\Pi_{h} \Psi^{n}-\Psi_{h}^{n}$. Subtracting (50) from (19), (51) from (20), and (52) from (21) and in the resulting equations using the test functions $w_{h}=\mu^{n}, v_{h}=\chi^{n}$, and $v_{h}=\zeta^{n}$ in the first, second, and third equations, respectively, we obtain the error equations

$$
\begin{gathered}
\left(\frac{\mu^{n}-\mu^{n-1}}{\Delta t}, \mu^{n}\right)+\left(\nabla \cdot \chi^{n}, \mu^{n}\right)=\left(\epsilon^{n}, \mu^{n}\right), \\
\left(\zeta^{n}, \chi^{n}\right)=\left(\mu^{n}, \nabla \cdot \chi^{n}\right),
\end{gathered}
$$


and

$$
\begin{aligned}
\left(\chi^{n}, \zeta^{n}\right)= & \left(\Pi_{h} \Psi^{n}-\hat{\Psi}^{n}, \zeta^{n}\right)+\left(K\left(\mathcal{P}\left(\rho_{h}^{n}\right) \zeta^{n}, \zeta^{n}\right)\right. \\
& -\left(\left(K\left(\mathcal{P}\left(\rho_{h}^{n}\right)-K\left(\rho^{n}\right)\right) \check{\Gamma}_{h}^{n}, \zeta^{n}\right)\right. \\
& +\left(K\left(\mathcal{P}\left(\rho_{h}^{n}\right)\right)\left(\Gamma_{h}^{n}-\check{\Gamma}_{h}^{n}\right), \zeta^{n}\right) .
\end{aligned}
$$

Here we have defined $\check{\Gamma}_{h}^{n}$ by

$$
\left(K\left(\rho^{n}\right)\left(\breve{\Gamma}_{h}^{n}-\Gamma^{n}\right), v_{h}\right)=0, \quad v_{h} \in V_{h} .
$$

Note we have also replaced $\Psi^{n}$ on the right side of (55) by its $L^{2}$ projection.

Combining (53) - (55), applying smoothness and boundedness assumptions on $K(\rho)$, superconvergence, and IIolder's inequality we obtain

$$
\begin{aligned}
\frac{1}{2 \Delta t}[ & \left.\left\|\mu^{n}\right\|^{2}-\left\|\mu^{n-1}\right\|^{2}\right]+\| K\left(\mathcal{P}\left(\rho_{h}^{n}\right)^{1 / 2} \zeta^{n} \|^{2}\right. \\
\leq & \left(\frac{\mu^{n}-\mu^{n-1}}{\Delta t}, \mu^{n}\right)+\| K\left(\mathcal{P}\left(\rho_{h}^{n}\right)^{1 / 2} \zeta^{n} \|^{2}\right. \\
\leq & \frac{1}{2}\left\|\mu^{n}\right\|^{2}+\frac{1}{2}\left\|\epsilon^{n}\right\|^{2}+\left(\Pi_{h} \Psi^{n}-\hat{\Psi}_{h}^{n}, \zeta^{n}\right) \\
& +\left(\left(K\left(\mathcal{P}\left(\rho_{h}^{n}\right)-K\left(\rho^{n}\right)\right) \check{\Gamma}_{h}^{n}, \zeta^{n}\right)+\left(K\left(\mathcal{P}\left(\rho_{h}^{n}\right)\right)\left(\breve{\Gamma}_{h}^{n}-\hat{\Gamma}_{h}^{n}\right), \zeta^{n}\right)\right. \\
\leq & C\left\|\mu^{n}\right\|^{2}+\delta\left\|\zeta^{n}\right\|^{2}+C\left[h^{2(k+2)}+\Delta t^{2}\right]
\end{aligned}
$$

where $\delta \leq K_{*}$. Here we have also used the fact that

$$
\left\|\mathcal{P}\left(\hat{\rho}_{h}^{n}\right)-\rho^{n}\right\| \leq C h^{2} .
$$

Multiplying (57) by $\Delta t$ and summing on $n, n=1,2, \ldots, N$ and applying Gronwall's Lemma, we see that

$$
\left\|\mu^{N}\right\|^{2}+\sum_{n=1}^{N} \Delta t\left\|K\left(\rho_{h}^{n}\right)^{1 / 2} \zeta^{n}\right\|^{2} \leq\left\|\mu^{0}\right\|^{2}+C\left(\Delta t^{2}+h^{2(k+2)}\right) .
$$

Using (58), Approximation Properties A, and the triangle inequality we deduce the following theroem:

Theorem 4. Let $V_{h}=V_{h}^{0}, \tilde{V}_{h}=\tilde{V}_{h}^{0}$, and $W_{h}=W_{h}^{0}$, that is, the $R T_{0}$ spaces defined on a tensor product grid, and define the triplet $\left(\rho_{h}^{n}, \Gamma_{h}^{n}, \Psi_{h}^{n}\right) \in W_{h}^{k} \times V_{h}^{k} \times \tilde{V}_{h}^{k}$ by (50) - (52). Take $\rho_{h}^{0}=\hat{\rho}_{h}(\cdot, 0)$. Then there exists a positive constant $C$, independent of $h$ such that

$$
\left\|\rho_{h}^{N}-\hat{\rho}_{h}^{N}\right\|+\left(\sum_{n=1}^{N} \Delta t\left\|K\left(\rho_{h}^{n}\right)^{1 / 2}\left(\hat{\Gamma}_{h}^{n}-\Gamma_{h}^{n}\right)\right\|^{2}\right)^{\frac{1}{2}} \leq C\left(h^{2}+\Delta t\right) .
$$

We now proceed as in Section 3, and let $\rho_{H}^{n}$ be defined by (50)-(52) with $h=H$. The proof is identical to that given above accept for the bounds on the terms $T^{*}$ and $T^{* *}$. These bounds can be modified by simply replacing $H^{k+1}$ by $H^{k+2}$; i.e., since $k=0$, we replace $H$ by $H^{2}$.

We obtain the following theorem: 
Theorem 5. Let $V_{h}=V_{h}^{0}, \tilde{V}_{h}=\tilde{V}_{h}^{0}$, and $W_{h}=W_{h}^{0}$ and define the one Newton correction triplet $\left(\tilde{\rho}_{h}^{n}, \tilde{\Gamma}_{h}^{n}, \tilde{\Psi}_{h}^{n}\right), \in W_{h}^{0} \times V_{h}^{0} \times \tilde{V}_{h}^{0}$ by (28) - (30). Assume that the Approximation Properties $A$ holds, and take $\tilde{\rho}_{h}^{0}=\hat{\rho}_{h}(\cdot, 0)$. Then there exists a positive constant $C$, independent of $h$ such that

$$
\begin{gathered}
\left\|\tilde{\rho}_{h}^{N}-\rho^{N}\right\|+\left(\sum_{n=1}^{N} \Delta t\left\|K\left(\rho_{H}^{n}\right)^{1 / 2}\left(\tilde{\Gamma}_{h}^{n}-\Gamma^{n}\right)\right\|^{2}\right)^{\frac{1}{2}} \\
\leq C\left(h+\Delta t+H^{3-d / 2}\right) .
\end{gathered}
$$

\section{Conclusions and EXTEnsions}

We remark that we even though we only considered the case $k=0$ in Section 4 , we have established superconvergence for the $R T_{k}$ spaces, $k \geq 0$, for the scheme (50)-(52). The two-level scheme with the Newton correction outlined in Section 3 could be extended to multiple levels with multiple corrections. We are currently investigating these possibilities. Computational results for the algorithms outlined here are also in progress.

We also remark that one may be able to improve the rate of convergence given in Theorem 5 for the lowest-order case by substituting $\mathcal{P}\left(\rho_{H}^{n}\right)$ and $\mathcal{P}\left(\tilde{\rho}_{h}^{n}\right)$ for $\rho_{H}^{n}$ and $\tilde{\rho}_{h}^{n}$. Preliminary theoretical results indicate that this may give superconvergence for $\tilde{\rho}_{h}^{n}$ at the centers of each cell of order $h^{2}+H^{4-d / 2}+\Delta t$.

\section{REFERENCES}

1. T. Arbogast, An error analysis for Galerkin approximations to an equation of mixed ellipticparabolic type, Technical Report TR90-33, Dept. of Comp, and Appl. Matll., Rice University, Houston, TX, 1990.

2. T. Arbogast, M. F. Wheeler, and I. Yotov, Mixed finite element methods on general geometry, in preparation.

3. J. Bear, Dynamics of fluids in porous media, Elsevier, New York, 1972.

4. F. Brezzi, J. Douglas Jr., and L. D. Marini, Two families of mixed finite elements for second order elliptic problems, Numer. Math. 47, pp. 217-223, 1985.

5. J. Douglas, Jr., and J. E. Roberts, Global estimates for mixed methods for second order elliptic equations, Math. Comp. 44, pp. 39-52, 1985.

6. R. E. Ewing, R. D. Lazarov, and J. Wang, Superconvergence of the velocity along the Gauss lines in mixed finite element methods, SIAM J. Numer. Anal. 28, pp. 1015-1029, 1991.

7. F. Milner, Mixed finite element methols for quasilinar second-order elliptic problems, Math. Comp. 44, pp. 303-320, 1985.

8. M. Nakata, A. Weiser, and M. F. Wheeler, Some superconvergence results for mixed finite element methods for elliptic problems on rectangular domains, in The Mathematics of Finite Elements and Applications V, J. R. Whiteman, ed., Academic Press, London, 1985.

9. J. C. Nedelec, Mixed finite elements in IR ${ }^{3}$, Numer. Math. 35, pp. 315-341, 1990.

10. P. A. Raviart and J. M. Thomas, A mixed finite element method for 2 nd order elliptic problems, in Mathematical Aspects of the Finite Element Method, Rome 1975, Lecture Notes in Mathematics, Springer-Verlag, Berlin, 1977.

11. A. Weiser and M. F. Wheeler, On convergence of block-centered finite differences for elliptic problems, SIAM J. Numer. Anal. 25, pp. 351-375, 1988.

12. J. Xu, Two-grid finite element discretization for nonlinear elliptic equations, preprint.

Department of Computational and applied Mathematics, Rice University, Houston, TX 77251

E-mail address: clint@rice.edu, mfw@rice.edu 